\title{
The Brønsted correlation for phenalene hydrocarbons
}

\author{
Andrew Streitwieser*, Michael J. Kaufman, Daniel A. Bors, Craig A. MacArthur, \\ James T. Murphy, and Francois Guibé \\ Department of Chemistry, University of California, Berkeley CA 94720-1460 \\ E-mail: astreit@berkeley.edu
}

Dedicated to Professor Eusebio Juaristi on the occasion of his $55^{\text {th }}$ birthday

(received 10 Mar 05; accepted 11 May 05; published on the web 14 May 05)

\begin{abstract}
Rate constants for tritium exchange in methanolic sodium methoxide are reported for phenalene, 1, 7-H-benzo[de]anthracene (benzanthrene), 2, 6-H-benzo[cd]pyrene (benzpyrene), 3, and 1,3diphenylpropene, $\mathbf{4}$, and compared with the corresponding relative ion pair $\mathrm{pK}$ values in THF. 4 is a vinylogous polyarylmethane and fits the previously established Brønsted correlation for polyarylmethanes. $\mathbf{2}$ and $\mathbf{3}$ are also part of this Brønsted family. The parent compound, phenalene is found to deviate from this correlation as well as a different Brønsted correlation found for hydrocarbons containing a cyclopentadienyl moiety. Phenalene is a unique member of still another Brønsted family.
\end{abstract}

Keywords: Brønsted correlation, kinetic acidity, ion pair acidity, carbon acidity, intrinsic barrier, tritium, hydrogen isotope exchange, base catalysis

\section{Introduction}

In a series of publications over several decades, we have presented kinetic results concerning the hydrogen isotope exchange reactivities of carbon acids, and compared these data with cesium ion-pair $\mathrm{pK}$ values obtained in cyclohexylamine (CHA). ${ }^{1-4}$ The compounds employed in these studies were hydrocarbon acids that yield highly delocalized carbanions - indene, fluorene, diand triphenylmethane, and related compounds. These studies showed two distinct Brønsted correlations. Compounds containing a cyclopentadiene moiety, including indenes and fluorenes, give a Brønsted correlation between kinetic acidities in $\mathrm{NaOMe} / \mathrm{MeOH}$ and equilibrium cesium ion pair acidities in CHA that is linear over a range of $10 \mathrm{pK}$ units. ${ }^{3}$ This correlation is distinctly different from that given by polyarylmethanes; the two Brønsted slopes are 0.37 and 0.58 , respectively, at $45^{\circ} \mathrm{C}$. In view of the similarity in delocalization for anionic stabilization among these compounds, it is curious that the two groups should produce such markedly different 
Brønsted correlations. However, an important difference between the two classes of compounds centers on the cyclopentadiene ring present in the first group; the additional $4 n+2$ aromaticity of such anions might well be responsible for the difference in behavior.

In order to test this theory, we extended the study to compounds containing a phenalene moiety. Phenalenyl anion is not a $4 n+2$ system but it does have conjugation of charge around a periphery that resembles that in cyclopentadienyl (eq. 1). In this paper we report such results for phenalene, 1, benzanthrene (7-H-benzo[d,e]anthracene ), 2, and benzpyrene (6-Hbenzo[c,d]pyrene), 3, and include acidity data for trans-1,3-diphenylpropene, 4, a modified arylmethyl system. We will also use the relative acidities of the cesium ion pairs in THF. ${ }^{5}$ For delocalized carbanions, ion pair pK's in CHA are known to correspond closely to the cesium ion pairs in THF. Accordingly, Brønsted correlations between the kinetic acidities in methanol and the cesium/THF ion pair pK's are essentially unchanged (vide infra).<smiles>C1=Cc2cccc3cccc(c23)C1</smiles>

1<smiles>c1ccc2c(c1)Cc1cccc3cccc-2c13</smiles>

2<smiles></smiles>

3<smiles>C(=C/c1ccccc1)\Cc1ccccc1</smiles>

4

Eq. 1

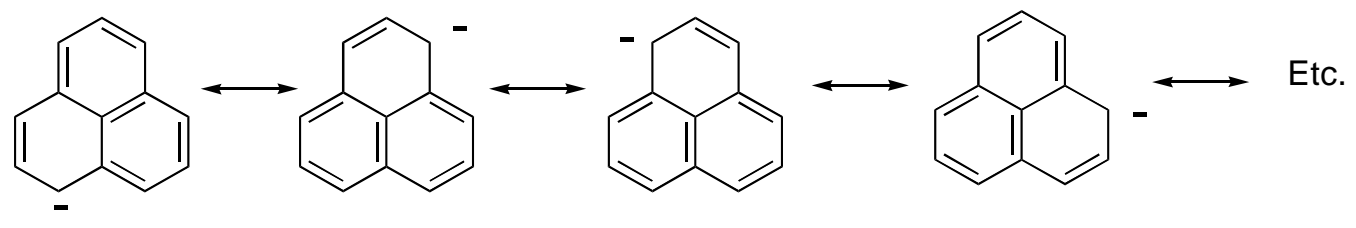

\section{Results and Discussion}

Equilibrium acidities. The equilibrium acidities of compounds 1-3 have been previously determined in CHA and are shown in Table $1 .{ }^{6}$ The acidity of $\mathbf{4}$ was measured in $\mathrm{THF}^{7}$ and is part of our table of cesium ion pair acidities in THF. ${ }^{5}$ The cesium salt of $\mathbf{4}$ has $\lambda_{\max }=533 \mathrm{~nm}$ ( $\varepsilon=53600)$, values close to those in THF, $\lambda_{\max }=538 \mathrm{~nm}(\varepsilon=57800)$. Results of several equilibrium acidity determinations with 4 in CHA are shown in Table 2. 
Table 1. Equilibrium ion pair acidities for cesium salts and rate constants for tritium exchange with $\mathrm{NaOMe}$

\begin{tabular}{lccc}
\hline \multicolumn{1}{c}{ Compound } & $\mathrm{pK}(\mathrm{CHA})^{\mathrm{a}}$ & $\mathrm{pK}(\mathrm{THF})$ & ${ }^{\mathrm{b}}{ }_{2}{ }^{\mathrm{OT}} \mathrm{M}^{-1} \mathrm{~s}^{-1}\left(45^{\circ} \mathrm{C}\right)$ \\
\hline 1, Phenalene & 18.49 & 18.52 & $1.6 \times 10^{-3}$ \\
2, Benzanthrene & 21.43 & 21.48 & $6.0 \times 10^{-4} \mathrm{e}$ \\
3, Benzpyrene & 19.91 & 19.95 & $3.0 \times 10^{-3}$ \\
4, 1,3-Diphenylpropene & $27.82^{\mathrm{c}}$ & $27.85^{\mathrm{d}}$ & $6.8 \times 10^{-5 \mathrm{f}}$ \\
\hline
\end{tabular}

${ }^{\text {a }}$ Ref. ${ }^{6}$

${ }^{\mathrm{b}}$ From $\mathrm{p} K_{\mathrm{CsCHA}}=1.0084 \mathrm{p} K_{\mathrm{Cs} / \mathrm{THF}}-0.13$ (Ref. ${ }^{5}$ ).

c This work.

${ }^{\mathrm{d}}$ Ref. $^{7}$

${ }^{\mathrm{e}} k_{2}{ }^{\mathrm{oT}}$ at $25^{\circ} \mathrm{C}, 6.0 \times 10^{-5} \mathrm{M}^{-1} \mathrm{~s}^{-1}$

${ }^{\mathrm{f}}$ At $98.5^{\circ} \mathrm{C}$.

Table 2. Equilibrium ion pair acidities of the cesium salt of 4 in CHA

\begin{tabular}{ccc}
\hline Indicator $^{\mathrm{a}}$ & $K^{b}$ & $\mathrm{p} K^{\mathrm{c}}$ \\
\hline BDPM & 93.1 & $28.20 \pm 0.50$ \\
PDDA & 1.48 & $27.84 \pm 0.06$ \\
PDDA & 1.52 & $27.83 \pm 0.05$ \\
PDDA & 1.67 & $27.79 \pm 0.07$ \\
PDDA & 1.64 & $27.80 \pm 0.11$ \\
BDDA & 0.82 & $27.81 \pm 0.05$ \\
BDDA & 0.74 & $27.85 \pm 0.05$ \\
\hline
\end{tabular}

a Indicator acids with pKs in $\mathrm{CHA}^{8}$ : BDPM, p-biphenylyldiphenylmethane, 30.17; PDDA, 10phenyl-9,9-dimethyl-9,10-dihydroanthracene, 28.01; BDDA, 10-p-biphenylyl-9,9-dimethyl-9,10dihydroanthracene, 27,72.

${ }^{\mathrm{b}} \mathrm{K}=[\mathrm{RCs}][$ Ind-H]/[RH][Ind-Cs]. Ind = Indicator.

${ }^{\mathrm{c}}$ Statistically corrected to a per-hydrogen basis.

The average value of 27.82 for the anion of $\mathbf{4}$ compares well with the value, 27.95, derived from the correlation published previously for the relation between the CHA and THF scales for cesium salts. ${ }^{5}$ This comparison lends confidence to the THF pKs assigned to 1-3 from their CHA acidities.

Kinetic Acidities. Rate constants for isotope exchange in methanolic sodium methoxide were obtained by monitoring the pseudo first-order decrease in specific activity from the tritiated substrate acids. The results of several experiments are summarized in Table 1 . The tabulated second-order rate constants $\left(\mathrm{k} 2^{\circ} \mathrm{T}\right)$ have been corrected to account for their dependence on base 
concentration. ${ }^{1}$ For phenalene and 1,3-diphenylpropene, the recorded $\mathrm{k} 2^{\circ} \mathrm{T}$ values have also been adjusted to account for the base-catalyzed scrambling of the tritium label into kinetically inactive positions (vida infra). It was quickly discovered during the course of these studies that the phenalene hydrocarbons, and particularly phenalene itself, are rapidly air-oxidized to the corresponding ketones in basic solution. This oxidation appears to be accelerated by light and necessitated that the kinetic runs be performed in an inert atmosphere under dim red lighting. When possible, the exchange reactions were monitored for several half-lives and data obtained after ca. ten half-lives were taken as infinity values. Of the two kinetic runs involving phenalene, an experimental infinity value could only be obtained in the run performed at high methoxide concentration. In the longer run conducted at a lower base concentration, kinetic aliquots obtained after ca. 60\% reaction contained large amounts of the oxidation product phenalenone. These aliquots were discarded and an infinity value was calculated from the remaining data. ${ }^{8}$ The residual tritium content was calculated to be $3.7 \%$ of the initial content, which is in satisfactory agreement with the experimental value of $6.4 \%$.

These exchange reactions have a substantial primary isotope effect, $\mathrm{k}_{\mathrm{H}} / / \mathrm{k}_{\mathrm{T}} \cdot{ }^{1}$ As a result, for $\mathbf{1}$ and $\mathbf{4}$ an equilibrium distribution of methylene- and olefin-labelled substrate is produced before the detritiation reaction has proceeded very far. Since the olefin-tritiated compound undergoes exchange at a rate several orders of magnitude slower than the methylene-labelled compound, ${ }^{9}$ the net effect of this equilibrium is to scramble the radiolabel into kinetically inert positions. This results in an experimental rate constant that is lower than the actual rate constant. Isotope scrambling in phenalene has been determined experimentally and is almost statistical. ${ }^{9}$ For 1,3-diphenylpropene the isotope distribution after equilibrium had been established was measured by ozonolysis and a reductive workup to give a mixture of tritiated 2-phenylethanol and benzyl alcohol which were separated by preparative gas chromatography. Since all of the labeled $\beta$-phenylethanol is derived from the 3-t isomer and all of the labeled benzyl alcohol results from the 1-t isomer, the equilibrium constant $K=3-t / 1-t$ is straightforward; $K=1.37 \pm$ 0.06. A second measurement after equilibration for a longer time gave $K=1.47 \pm 0.08$, an agreement within experimental error of the previous result. We used the average, $K=1.42$, to correct the kinetic results. 


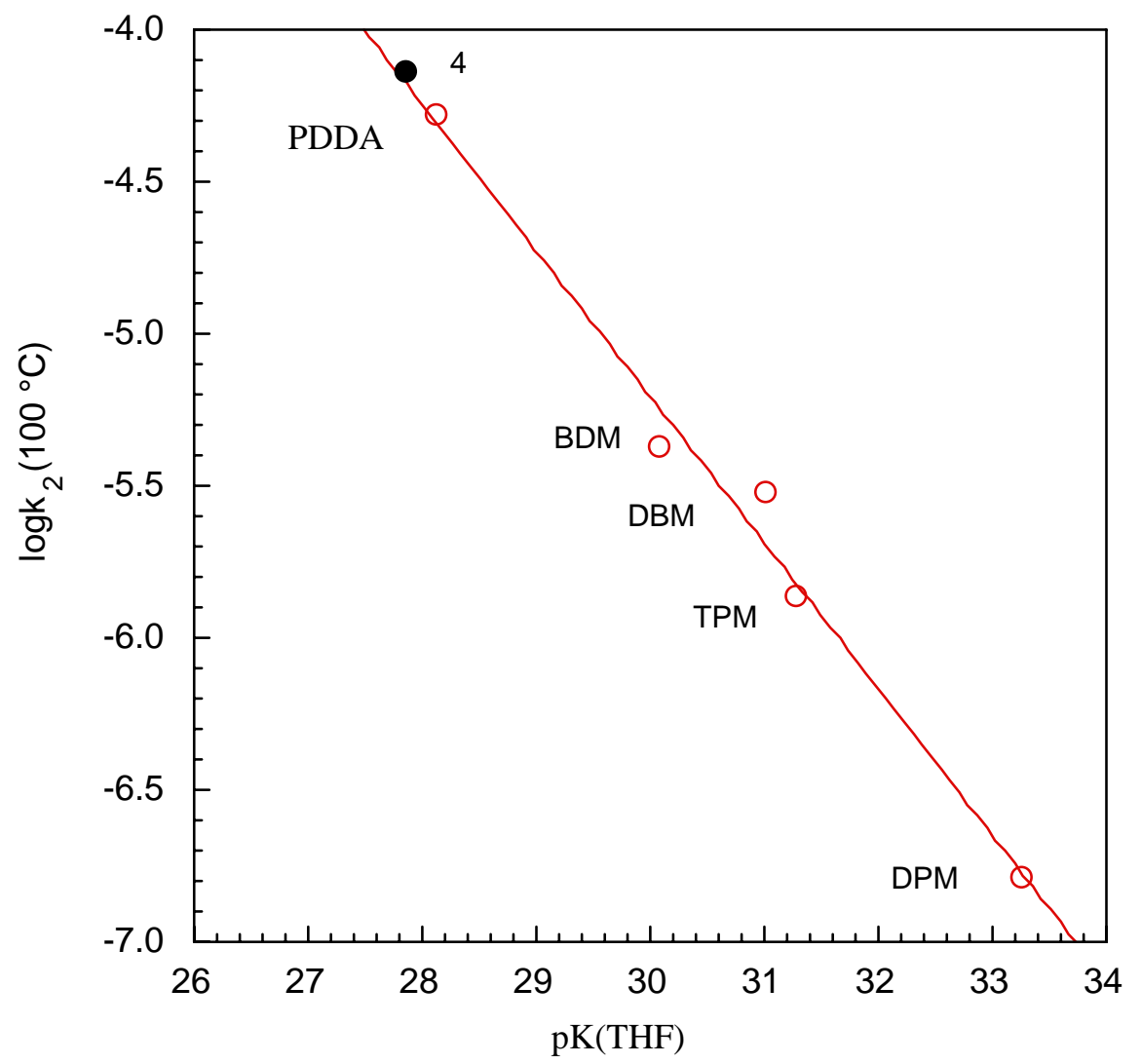

Figure 1. Compound 4 (black circle) fits the Brønsted correlation given by five polyarylmethanes at $100{ }^{\circ} \mathrm{C}$. Compounds in red are: PDDA, 10-phenyl-9,9-dimethyl-9,10dihydroanthracene; BDM, biphenylyldiphenylmethane; DBM, dibiphenylylmethane; TPM, triphenylmethane; DPM, diphenylmethane. The Brønsted slope (in red) given without 4 is $0.48 \pm$ $0.03 ; \mathrm{R}^{2}=0.985$.

Compound 4 was run at $98.5{ }^{\circ} \mathrm{C}$ and is compared in Figure 1 with a series of polyarylmethanes at $100{ }^{\circ} \mathrm{C}$ that form a linear Brønsted plot. ${ }^{2}$ For this purpose the rate of $\mathbf{4}$ was increased by $7 \%$ (corresponding to an Arrhenius $\mathrm{E}^{*}$ of $28 \mathrm{kcal} \mathrm{mol}^{-1}$ ) to account for the $1.5^{\circ} \mathrm{C}$ temperature difference. It is clear that $\mathbf{4}$, a vinylogous polyarylmethane, belongs firmly in this Brønsted family.

The other comparisons are all made at $45^{\circ} \mathrm{C}$. The combined Brønsted correlations for the fluorene, polyarylmethane, and phenalene acids are shown in Figure 2. Two features of these plots are noteworthy. First, it is clear that the phenalene derivatives $\mathbf{2}$ and $\mathbf{3}$ form an extension of the polyarylmethane correlation, although $\mathbf{3}$ is so close to the intersection of both correlations that it could be a member of either. It is equally clear that phenalene is not a member of either of the correlations. The significance of these results will now be discussed. 


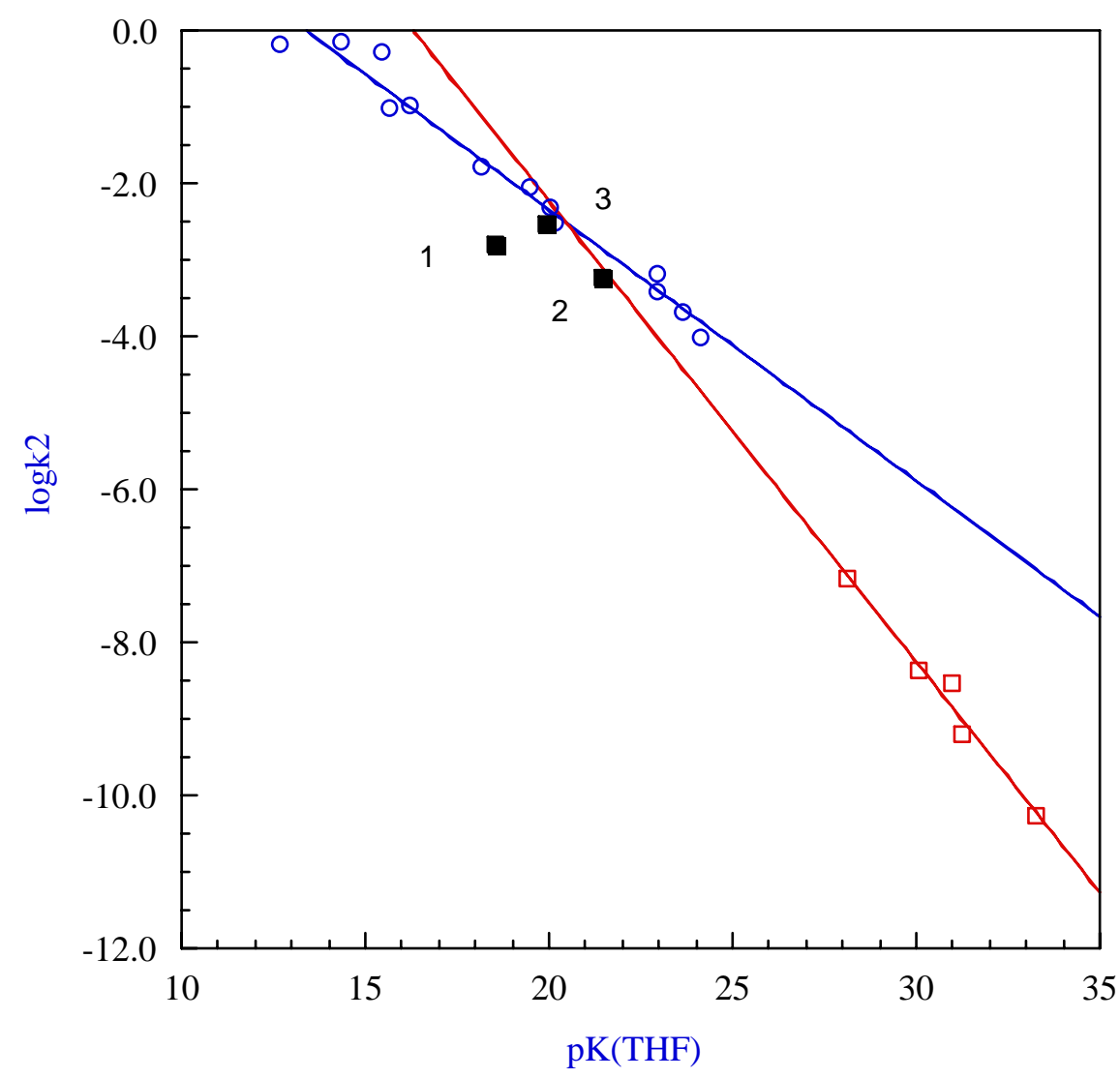

Figure 2. Two Brønsted correlations at $45{ }^{\circ} \mathrm{C}$. The polyarylmethanes (red) are $9.81 \pm 1.84-$ $(0.60 \pm 0.06) x ; R^{2}=0.971$. Fluorenes, indenes, etc. (blue) are $4.73 \pm 0.34-(0.35 \pm 0.02) x ; R^{2}=$ 0.973. The phenalenes $\mathbf{1}, \mathbf{2}$, and $\mathbf{3}$, are solid black squares.

Phenalene Derivatives. The equilibrium acidities of benzanthrene, 2, and benzpyrene, 3, are greater than those of the polyarylmethane acids by a factor of $10^{6}-10^{12}$. Despite these large differences in acidities, both of the phenalene derivatives fit remarkably well onto the Brønsted correlation for polyarylmethane acids. This result can be attributed to the structural and electronic similarities shared by both groups of compounds. In both the phenalene and polyarylmethane acids, proton abstraction occurs from a carbon center adjacent to two or more phenyl groups to yield a highly delocalized carbanion. The long range of linearity of the Brønsted correlation for these acids can then be explained on the basis of two contributing factors. First, the fact that large acidity differences result from rather modest structural perturbations allows us to compare carbon acids for which reactivity differences due to steric factors are minimized. Secondly, the extensive degree of electronic reorganization which accompanies carbanion formation results in a large energy barrier for proton transfer. A direct consequence of such a large intrinsic barrier is a relatively long range of linearity in the Brønsted correlation. ${ }^{10,11}$ 
It can be seen from the Brønsted correlations shown in Figure 2 that benzanthrene is slightly less reactive than a fluorene-type acid of comparable equilibrium acidity. The phenalene and polyarylmethane acids derive their acidic properties from the extensive delocalization of charge in the derived anions. The fluorene acids contain a cyclopentadienyl moiety which leads to an aromatic system upon deprotonation. Our results with the phenalene derivatives demonstrates that the cyclopentadienyl moiety is more efficient at delocalizing negative charge. For the aromatic-stabilized acids, a greater amount of charge is delocalized for a given degree of proton transfer, and the transition state is reached at a relatively early stage compared to the polyarylmethane acids. This analysis is consistent with the magnitude of the Brønsted exponents for the two correlations, 0.35 for the fluorenes and 0.60 for the polyarylmethanes. The classical interpretation of the Brønsted exponent as a measure of the degree of proton transfer at the transition state has been criticized since the discovery that some reaction series give values outside the limits of zero to unity. However, the systems for which anomalous exponent values have been found involve substrate acids in which a nearby substituent may exert different inductive effects upon the transition state and final state charge distributions. The mechanism of anionic stabilization for the compounds employed in our own studies is primarily charge delocalization. For these conjugated hydrocarbon acids, the value of the Brønsted exponent is likely to be a valid measure of the degree of charge delocalization at the transition state.

Phenalene itself, however, is about ten times less reactive than a member of either group with comparable acidity. It seems likely that the proper view is that its equilibrium acidity is much greater than its kinetic acidity would indicate based on the other systems. The conjugate anion is a symmetrical system with extensive delocalization but at the transition state for deprotonation the partial charge generated is that of a benzylic system with conjugation to a naphthalene ring; that is, achieving the stabilization inherent in the complete carbanion means giving up the naphthalene resonance, a feature that probably gives rise to the relatively high kinetic barrier. As a result, phenalene finds itself the unique member of a new Brønsted family.

\section{Experimental Section}

General Procedures. Unless otherwise noted chemicals were obtained from commercial suppliers and were purified by recrystallization and/or sublimation if solid or distillation if liquid. Diethyl ether and tetrahydrofuran (THF) were distilled from dark purple solutions of the solvent and sodium/benzophenone just prior to use. All indicator acids for the equilibrium acidity measurements were recrystallized at least twice from ethanol, then sublimed under high vacuum. Methanol used for kinetics was distilled from fresh magnesium metal and degassed by repeated freeze/thaw cycles prior to use. IR spectra were determined with a Perkin-Elmer Model 337 Grating Infrared Spectrometer. Reactions involving organometallic reagents were performed under an inert atmosphere. To minimize air oxidation the phenalene hydrocarbons 
were handled under an argon atmosphere and were stored under argon in the dark when not in use.

Compound characterization and kinetics. Phenalene, benzanthrene (benzo[cd]anthracene), and benzo[cd]pyrene were available from a previous study. ${ }^{6}$

Phenalene-t. To a solution of $0.281 \mathrm{~g}$ (1.69 mmol) of phenalene in $25 \mathrm{~mL}$ of THF was added $1 \mathrm{~mL}$ of a $1.7 \mathrm{M}$ solution of n-butyllithium in hexane. The solution was swirled briefly, and quenched with $1 \mathrm{~mL}$ of tritiated methanol (activity $5.4 \times 10^{9} \mathrm{dpm} / \mathrm{mL}$ ). The solution was poured into $20 \mathrm{~mL}$ of water, and the organic phase was separated and dried. Removal of the solvent followed by sublimation of the residue gave $0.195 \mathrm{~g}(69.4 \%)$ of a white solid, $\mathrm{mp} 82-83^{\circ} \mathrm{C}$. The mass spectrum was identical to that of an unlabelled sample. The specific activity of the compound was $1.12 \times 10^{6} \mathrm{dpm} / \mathrm{mg}$. A second preparation had $6.8 \times 10^{4} \mathrm{dpm} / \mathrm{mg}$.

Benzo[cd]anthracene-7-t. To a solution of $1.50 \mathrm{~g}(6.93 \mathrm{mmol})$ of the hydrocarbon and $100 \mathrm{~mL}$ of THF was added $0.120 \mathrm{~mL}$ of $5.1 \mathrm{M}$ sodium methoxide and $2.0 \mathrm{~mL}$ of methanol(t) (activity 7.2 x $10^{6} \mathrm{dpm} / \mathrm{mL}$ ). This solution was stirred for $2 \mathrm{~h}$, then quenched with $0.5 \mathrm{~mL}$ of glacial acetic acid. The quenched solution was extracted with ether $(2 \times 100 \mathrm{~mL})$, and the ether layers were washed with water (5 x $100 \mathrm{~mL})$ and saturated aqueous $\mathrm{NaHCO}_{3}(1 \times 100 \mathrm{~mL})$. After drying over $\mathrm{Na}_{2} \mathrm{SO}_{4}$ rotary evaporation afforded a white residue which was recrystallized twice from alcohol. Sublimation gave $0.50 \mathrm{~g}$ (33\%) of a white solid with physical and spectral properties in accord with the unlabelled hydrocarbon. The activity of the sample was $1.1 \times 10^{6} \mathrm{dpm} / \mathrm{mg}$.

6-H-Benzo[cd]pyrene-6-t. To a solution of $30 \mathrm{mg}(0.125 \mathrm{mmol})$ of the hydrocarbon in $8 \mathrm{~mL}$ of diethyl ether was added one equivalent of a $1.7 \mathrm{M}$ hexane solution of n-butyllithium. The solution was swirled briefly, and $50 \mathrm{mg}(0.272 \mathrm{mmol})$ of $\mathrm{MgBr}_{2}$ in $10 \mathrm{~mL}$ of ether was added. The Grignard reagent was then quenched with $1.0 \mathrm{~mL}$ of tritiated water. More water was added, and the product was extracted with ether. The ether layer was washed several times with water and dried over $\mathrm{MgSO}_{4}$. After evaporation of the ether, tbe crude product was chromatographed on alumina (2:1 v/v hexane-benzene eluent) to give $20 \mathrm{mg}(67 \%)$ of tritiated benzo[cd]pyrene, specific activity 1.54 × $10^{6} \mathrm{dpm} / \mathrm{mg}$.

trans-1,3-Diphenylpropene. The method of Stoermer, Thiel, and Laage was used with modification. ${ }^{12}$ In a round bottom flask equiped with a reflux condenser was combined $15.0 \mathrm{~g}$ $(0.267 \mathrm{~mol})$ of $\mathrm{KOH}$ pellets and $100 \mathrm{~mL}$ of absolute ethanol. To this solution was added $30.2 \mathrm{~g}$ $(0.251 \mathrm{~mol})$ of phenylacetaldehyde in one portion. The resulting intense red solution was stirred at reflux for $4 \mathrm{~h}$ then cooled to room temperature. The solution was diluted with $500 \mathrm{~mL}$ of water and extracted with $200 \mathrm{~mL}$ of ether. The aqueous phase was re-extracted with a fresh $150 \mathrm{~mL}$ portion of ether, and the combined ether layers were washed with $10 \%$ aq. $\mathrm{NaOH}(1 \times 250 \mathrm{~mL})$ and water $\left(1 \times 250 \mathrm{~mL}\right.$ ). The organic extract was dried over $\mathrm{MgSO}_{4}$ and the solvent was removed on a rotary evaporator. The residue consisted of an orange oil which was homogenous by TLC. The product was decolorized by chromatography on silica gel (hexane eluent), yielding $14.9 \mathrm{~g}$ (61\%) of a thin colorless oil. The trans geometry was assigned on the basis of comparison with 
published data. IR (thin film): 3040, 1595, 960,738, $697 \mathrm{~cm}^{-1} ;{ }^{1} \mathrm{H}-\mathrm{NMR}\left(\mathrm{CDCl}_{3}\right): \delta 3.5$ (d, $\left.2 \mathrm{H}\right)$, 6.3-6.5 (m, 2H), 7.2-7.4 (m, 10H). UV (hexane): $253 \mathrm{~nm}$ (26200).

trans-1,3-Diphenylpropene-3-t. The labelling procedure was identical to that already described for phenalene. The yield of labelled substrate was $76 \%$.

Kinetics. The procedure used to obtain kinetic data has been previously documented. ${ }^{1,2}$ The measurements of $\mathbf{2}$ and $\mathbf{3}$ were unexceptional. Phenalene, however, presents some complications. One is the fact that the facile oxidation of phenalene required a high concentration of the sodium methoxide base catalyst in order to shorten the reaction time. The concentration used, about $1 \mathrm{M}$, required a "salt effect" correction which was determined from the original data on fluorene. ${ }^{1} \mathrm{~A}$ least squares fit to a second order polynomial more accurately represents the catalytic effect of $\mathrm{NaOMe}$ at higher concentrations (eq. 2)

$\mathrm{k}_{2}^{\circ}=\mathrm{k}_{2} /\left(1+0.167[\mathrm{NaOMe}]+0.0324[\mathrm{NaOMe}]^{2}\right)$ at $45^{\circ} \mathrm{C}\left(\mathrm{R}^{2}=0.9997\right)$

Another correction that must be applied results from the scrambling of the tritium label into non-reactive positions in the molecule. Tritium isotope effects are rather large and it is a good assumption that the equilibrium in eq 3 is established early in the kinetic run.

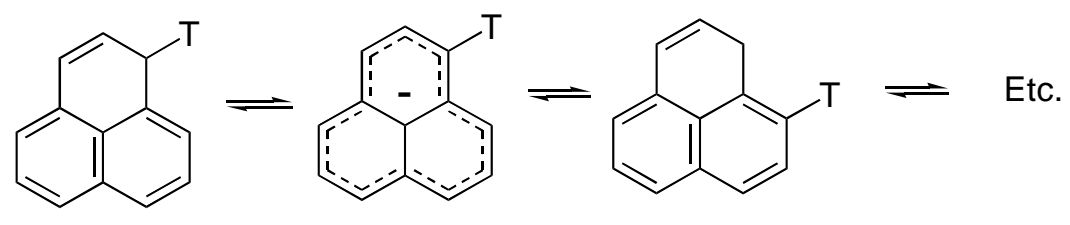

The correction can be estimated quite accurately because deuterium scrambling in phenalene has been examined experimentally. ${ }^{9}$ We assume only that the deuterium content in the olefinic position is the same as the aromatic positions to give the fraction of deuterium in the reactive position as $0.304 \pm 0.003$, a value only slightly different from the value of $2 / 7=0.286$. That is, there is only a slight secondary deuterium isotope. Converting this deuterium isotope effect into the corresponding tritium isotope effect ${ }^{1}$ gives the fraction of total tritium in the reactive position as 0.312 .

The isotope exchange kinetics were determined by removing aliquots from the methanolic $\mathrm{NaOMe}$ solution with an argon-flushed syringe and quenching with aqueous $\mathrm{HCl}$. The solution was extracted with cyclohexane, and the organic layer was dried over $\mathrm{MgSO}_{4}$. One aliquot was diluted with the scintillation fluid and counted on a scintillation counter. Another portion was used to measure the absorbance at $343 \mathrm{~nm}$ and to monitor the decomposition of the phenalene. None of the kinetic points including the infinity point showed the distinctive peak at 352 and 377 $\mathrm{nm}$ for phenalone. The aliquots were placed on an equimolar basis by dividing the dpm by the relative concentration. The data are summarized in Table 3 and plotted in Figure 3. 
Table 3. Protodetritiation of phenalene at $45^{\circ} \mathrm{C}$ with $1.093 \mathrm{M} \mathrm{NaOMe}$ in methanol

\begin{tabular}{cc}
\hline Time, $\mathrm{s}$ & $\mathrm{dpm}^{\mathrm{a}}$ \\
\hline 328 & 7491 \\
908 & 5938 \\
1941 & 2126 \\
2661 & 1589 \\
3637 & 1089 \\
4479 & 917.9 \\
18278 & 510.6 \\
\hline
\end{tabular}

${ }^{a}$ Disintegrations per minute on an equimolar basis.

The pseudo-first order rate constant of $7.21 \mathrm{E}-4 \mathrm{~s}^{-1}$ is divided by [NaOMe] to give the second order rate constant and by 0.312 to correct for redistribution of tritium. Application of eq. 1 gives the final $k_{2}{ }^{\mathrm{oT}}=1.73 \mathrm{E}-3 \mathrm{M}^{-1} \mathrm{~s}^{-1}$. A second run with $[\mathrm{NaOMe}]=0.124$ gave $k_{2}{ }^{\mathrm{TT}}=$ 1.49E-3 $\mathrm{M}^{-1} \mathrm{~s}^{-1}$. We took the average for Table 1 and Figure 2.

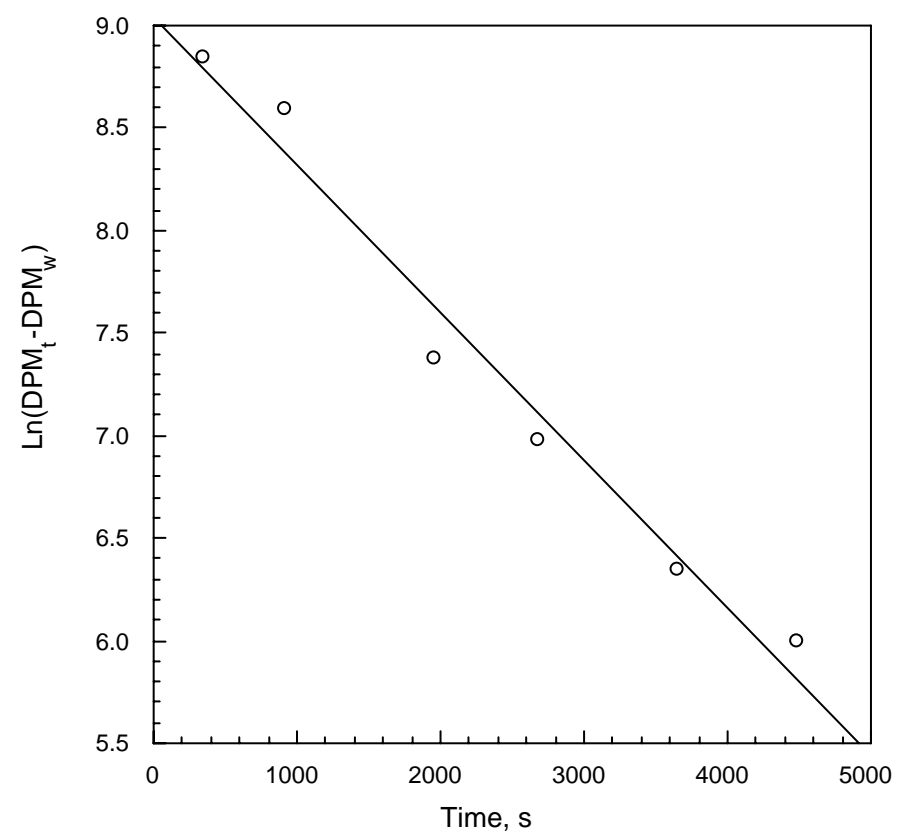

Figure 3. Protodetritiation of phenalene in 1.093M NaOMe at $45^{\circ} \mathrm{C}$. Slope gives first order rate constant $=(7.21 \pm 0.59) \mathrm{E}-4 \mathrm{~s}^{-1} ; \mathrm{R}^{2}=0.974$.

\section{Acknowledgements}

This research was supported in part by grants from the National Science Foundation. 


\section{References}

1. Streitwieser, A. Jr.; Hollyhead, W.; Pudjaatmaka, A.; Owens, P. H.; Kruger, T.; Rubenstein, P.; MacQuarrie, R.; Brokaw, M.; Chu, W.; Niemeyer, H. M. J. Am. Chem. Soc. 1971, 93, 5088.

2. Streitwieser, A. Jr.; Hollyhead, W.; Sonnichsen, G.; Pudjaatmaka, A.; Chang, C. J.; Kruger, T. J. Am. Chem. Soc. 1971, 93, 5096.

3. Streitwieser, A. Jr.; Kaufman, M. J.; Bors, D. A.; Murdoch, J. R.; MacArthur, C. A.; Murphy, J. T.; Shen, C. C. J. Am. Chem.Soc. 1985, 107, 6983.

4. Streitwieser, A. A Life with the Synergy of Theory and Experiment; American Chemical Society: Washington DC, 1996; p 85.

5. Streitwieser, A.; Ciula, J. C.; Krom, J. A.; Thiele, G. J. Org. Chem. 1991, 56, 1074.

6. Streitwieser, A. Jr.; Word, J. M.; Guibe, F.; Wright, J. S. J. Org. Chem. 1981, 46, 2588.

7. Thiele, G.; Streitwieser, A. J. Am. Chem. Soc. 1994, 116, 446.

8. Streitwieser, A. Jr.; Juaristi, E.; Nebenzahl, L. L. In Comprehensive Carbanion Chemistry; Buncel, E.; Durst, T., Eds; Elsevier: 1980; Ch. 7.

9. Paquette, L. A.; Gree, R. J. Organometal. Chem. 1978, 146, 319.

10. Marcus, R. A. J. Phys. Chem. 1968, 72, 891.

11. Murdoch, J. R. J. Am. Chem. Soc. 1972, 94, 4410.

12. Stoermer, R.; Thiel, C.; Laange, E. Chem. Ber. 1925, B58, 2607. 